\title{
Efficient High-Gain Two-Crystal Optical Parametric Oscillator
}

\author{
Gerald T. Moore and Karl Koch
}

\begin{abstract}
A high-gain optical parametric oscillator containing two crystals in series with $90 \%$ signal outcoupling after the first crystal and $100 \%$ idler outcoupling after both crystals is found to give stable highly efficient operation over a wide dynamic range of pump intensity. Most of the small-signal gain results from the longer second crystal, but most of the energy conversion at saturation occurs in the shorter first crystal. Power loading in the crystals is predominately due to the incident pump, and is small in the second crystal after saturation. Theoretical description of the device is presented in terms of a plane-wave model, as well as a numerical model which incorporates transverse effects in one transverse dimension. It is found that the device can exhibit a period-two limit cycle when the two crystals are tuned to the same frequencies. Steady-state operation is obtained by tuning the crystals to slightly different frequencies, without significantly affecting the average efficiency. Experiments using KTP crystals synchronously pumped at $1.064 \mu \mathrm{m}$ by a $Q$-switched mode-locked Nd:YAG laser are proposed. For degenerate down-conversion, a calculation including transverse effects shows that $88.8 \%$ of the incident power is converted to $2.128 \mu \mathrm{m}$ in a single beam outcoupled after the first crystal. An additional 5.7\% emerges as idler power after the second crystal.
\end{abstract}

\section{INTRODUCTION}

$\mathbf{I}$ $\mathrm{N}$ order to achieve high intensities, optical parametric oscillators (OPO's) are usually pumped by pulsed lasers. Even cw-pumped OPO's have a variation in pump intensity as a function of the transverse coordinates. Unfortunately, the nonlinear dynamics of the conventional OPO leads to high conversion efficiency only for special values of the pump intensity. Very high efficiency is impossible when the pump intensity has large variations as a function of time or of the transverse coordinates. Recently [1], [2], we studied the dynamics of an OPO with intracavity sum-frequency generation (SFG) of the pump and signal. We found that highefficiency up-conversion to the sum frequency is possible in this device over a wide dynamic range of pump intensity, particularly when the SFG crystal precedes the OPO crystal in the cavity. In this SFG-OPO device, only two (the pump and signal) of the three fields coupled by the nonlinear interactions in each crystal are nonzero at the crystal entrances, so that

Manuscript received October 1, 1993; revised November 29, 1994. This work was supported in part by the U.S. Air Force Office of Scientific Research.

G. T. Moore is with the Nonlinear Optics Center of Technology, Phillips Laboratory/LIDN, 3550 Aberdeen Avenue, SE, Kirtland Air Force Base, NM 87117 USA. He is also with the Center of Advanced Studies, University of New Mexico, Alburquerque, NM 87131 USA.

K. Koch is with the Nonlinear Optics Center of Technology, Phillips Laboratory/LIDN, 3550 Aberdeen Avenue, SE, Kirtland Air Force Base, NM 87117 USA.

IEEE Log Number 9409849.

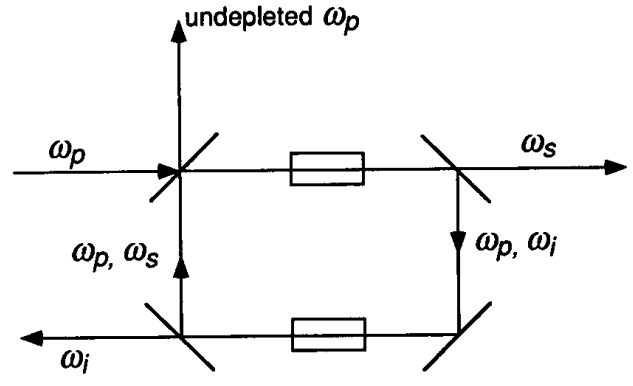

Fig. 1. Schematic diagram of the nonresonant two-crystal OPO.

interference effects sensitive to the relative phases of the fields are eliminated.

Our studies of the SFG-OPO device lead us here to consider whether the OPO in its traditional role of frequency downconversion $\left(\omega_{p} \rightarrow \omega_{s}+\omega_{i}\right)$ can be made more efficient by using two OPO crystals in series. Again the idea is to eliminate interference effects by having only two of the three coupled fields nonzero at the crystal entrances.

Two configurations are possible in which the two crystals both contribute to deplete the pump. One of these is a nonresonant configuration in which the idler is completely outcoupled after one crystal and the signal is completely outcoupled after the second crystal (see Fig. 1). Devices of this type have been operated using BBO [3] and KTP [4] crystals. In these devices the pump was retroreflected at one end of the cavity, and the signal and idler were outcoupled at opposite ends of the cavity. Thus, the "first" and "second" crystals were the same, though two crystals in series with reversed directions of walk-off were used. The KTP device was injection seeded, and achieved $36 \%$ efficiency and good beam quality in conversion of $0.532 \mu \mathrm{m}$ light to $0.760 \mu \mathrm{m}$ and $1.77 \mu \mathrm{m}$. However, our studied have shown that the nonresonant configuration tends to produce inefficient and very unstable dynamics, and we discuss it only briefly in this paper.

The configuration we treat here is a singly resonant device in which the signal is resonated and the idler is completely outcoupled after each crystal (see Fig. 2). We call this the OPO-OPO device. In this paper we consider the signal to be the field which is resonated, irrespective of whether the signal or idler has the higher frequency.

Let the two crystals have lengths $L_{a}$ and $L_{b}$, where the pump is incident first on crystal $a$. After crystal $a$ suppose that a fraction $R_{a}$ of the signal intensity is passed on to crystal $b$, 




Fig. 2. Schematic diagram of the singly resonant two-crystal OPO (the OPO-OPO device).

while a fraction $1-R_{a}$ is outcoupled. Similarly, after crystal $b$ suppose that a fraction $R_{b}$ of the signal intensity is passed on to crystal $a$, while a fraction $1-R_{b}$ is outcoupled. For practical applications it is desirable to outcouple the signal in only one place, in which case either $R_{a}=1$ or $R_{b}=1$. However, we present the analysis for the general case.

\section{Pump-Depleted Steady States}

We first consider a simple description of the problem that assumes phase-matched plane waves in both crystals. The coupled signal, idler, and pump waves can be described in terms of real amplitudes $\rho_{j}(z), j=s, i$, or $p$, obeying

$$
\begin{gathered}
\frac{d \rho_{s}}{d z}=\kappa \rho_{p} \rho_{i}, \\
\frac{d \rho_{i}}{d z}=\kappa \rho_{p} \rho_{s}, \\
\frac{d \rho_{p}}{d z}=-\kappa \rho_{s} \rho_{i},
\end{gathered}
$$

where the coupling constant $\kappa$ is given in terms of the refractive indices $n_{j}$ and the effective nonlinear coefficient $d$ by

$$
\kappa=\frac{d}{c} \sqrt{\frac{\omega_{s} \omega_{i} \omega_{p}}{n_{s} n_{i} n_{p}}}
$$

In general, $d$ and $n_{j}$ are different in the two crystals, so that $\kappa$ is replaced by either $\kappa_{a}$ or $\kappa_{b}$. The quantities $\rho_{j}^{2}(z)$ are proportional to the photon flux densities, and the intensities are given by $I_{j}=\frac{1}{2} c \epsilon_{0} \omega_{j} \rho_{j}^{2}$. To obtain high efficiency the pump must be highly depleted, so it is of interest to calculate the steady states of the device which completely deplete the pump. Let $z_{1}$ and $z_{2}$ be the entrance and exit of crystal $a$, and let $z_{3}$ and $z_{4}$ be the entrance and exit of crystal $b$. The flux densities $\rho_{j}^{2}\left(z_{k}\right)$ for the states of interest can be written as functions of a parameter $m_{b}$ defined below. They are determined up to a multiplicative constant $\beta^{2}$ by the conditions $\rho_{p}^{2}\left(z_{4}\right)=0, \rho_{s}^{2}\left(z_{3}\right)=R_{a} \rho_{s}^{2}\left(z_{2}\right), \rho_{s}^{2}\left(z_{1}\right)=R_{b} \rho_{s}^{2}\left(z_{4}\right)$, and the Manley-Rowe conserved quantities

$$
C_{s a}=\rho_{p}^{2}(z)+\rho_{s}^{2}(z)
$$

$$
C_{i a}=\rho_{p}^{2}(z)+\rho_{i}^{2}(z)
$$

in crystal $a$ and

$$
\begin{aligned}
& C_{s b}=\rho_{p}^{2}(z)+\rho_{s}^{2}(z), \\
& C_{i b}=\rho_{p}^{2}(z)+\rho_{i}^{2}(z),
\end{aligned}
$$

in crystal $b$. If we define $m_{a}=C_{i a} / C_{s a}$ and $m_{b}=C_{i b} / C_{s b}$, then it is easily shown that the nonzero flux densities at the crystal entrances and exits are given by

$$
\begin{gathered}
\rho_{p}^{2}\left(z_{1}\right)=\beta^{2}\left[1-\left(1-R_{a}\right) m_{b}-R_{a} R_{b}\right], \\
\rho_{s}^{2}\left(z_{1}\right)=\beta^{2} R_{a} R_{b}, \\
\rho_{p}^{2}\left(z_{2}\right)=\rho_{p}^{2}\left(z_{3}\right)=\rho_{i}^{2}\left(z_{4}\right)=\beta^{2} R_{a} m_{b}, \\
\rho_{s}^{2}\left(z_{2}\right)=\beta^{2}\left(1-m_{b}\right), \\
\rho_{i}^{2}\left(z_{2}\right)=\beta^{2}\left(1-m_{b}-R_{a} R_{b}\right), \\
\rho_{s}^{2}\left(z_{3}\right)=\beta^{2} R_{a}\left(1-m_{b}\right), \\
\rho_{s}^{2}\left(z_{4}\right)=\beta^{2} R_{a} .
\end{gathered}
$$

Equations (9) and (10) also imply that

$$
m_{a}=1-\frac{R_{a} R_{b}}{1-\left(1-R_{a}\right) m_{b}}
$$

so that (9) can also be written as

$$
\rho_{p}^{2}\left(z_{1}\right)=\beta^{2} R_{a} R_{b} \frac{m_{a}}{1-m_{a}} .
$$

Since (13) should be nonnegative, we have $0 \leq m_{b} \leq$ $1-R_{a} R_{b}$.

It is useful to introduce dimensionless coupling parameters $g_{a}=\kappa_{a} \rho_{p}\left(z_{1}\right) L_{a}$ and $g_{b}=\kappa_{b} \rho_{p}\left(z_{1}\right) L_{b}$ for the two crystals, and it is sufficient to take $\rho_{p}\left(z_{1}\right)$ positive. Though not essential [1], we assume for simplicity that there is no recollimation of the beams between the crystals. The net small-signal gain per pass is given by $G=G_{a} R_{a} G_{b} R_{b}$, where $G_{a}=\cosh ^{2} g_{a}$ and $G_{b}=\cosh ^{2} g_{b}$. As the pump intensity varies, as in pulsed operation or transversely across the beam, OPO operation takes place along a straight line through the origin in the $\left(g_{a}, g_{b}\right)$ space. In order to obtain high efficiency, we want to choose the slope of this operating line such that the line lies close to states with complete pump depletion over an extended range.

The solution of (1)-(3) for the pump field in the second crystal is

$$
\rho_{p}(z)=\sqrt{C_{i b}} \operatorname{sn}\left(K_{b}-\kappa_{b} \sqrt{C_{s b}}\left(z-z_{3}\right) \mid m_{b}\right),
$$

where sn denotes the Jacobi elliptic function. The quarterperiod $K_{b}=K\left(m_{b}\right)$ of this function is given by the complete elliptic integral of the first kind. We see from (15) that 


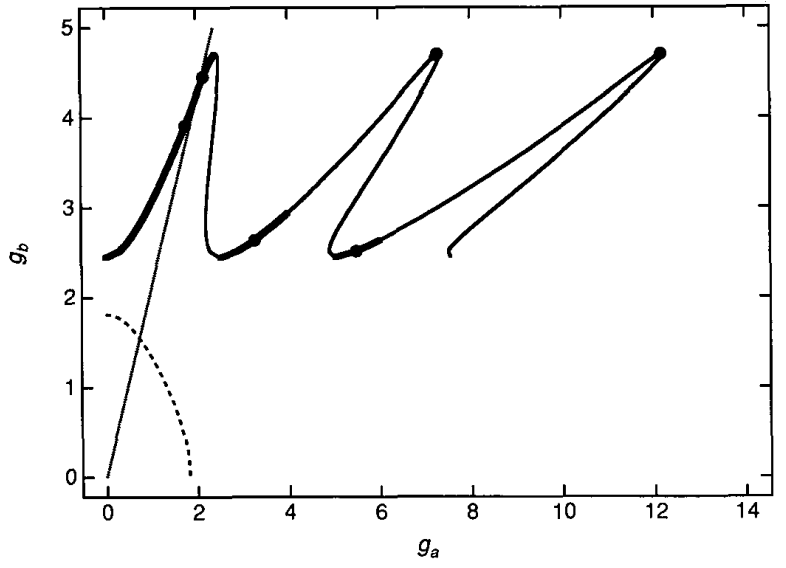

Fig. 3. Pump-depleted steady states for $n_{b}=1, R_{a}=0.1$, and $R_{b}=1.0$. Thick lines show the stable steady states. Filled circles indicate places where the stability parameter changes sign. Dashed line is the small-signal threshold. Dotted line of slope 2.1 is our choice of operating line.

$\sqrt{C_{s b}}=\beta \sqrt{R_{a}}$, where we take $\beta$ to be positive. In order that (18) vanish at the end of the second crystal, it is necessary that

$$
\kappa_{b} L_{b} \beta \sqrt{R_{a}}=\left(2 n_{b}-1\right) K_{b}
$$

where $n_{b}$ is a positive integer. If we combine this result with the square root of (17), we obtain

$$
g_{b}=\left(2 n_{b}-1\right) K_{b} \sqrt{\frac{R_{b} m_{a}}{1-m_{a}}} .
$$

The solution of (1)-(3) for the signal field in the first crystal is

$$
\rho_{s}^{2}(z)=C_{s a} \mathrm{dn}^{2}\left(K_{a}-\kappa_{a} \sqrt{C_{s a}}\left(z-z_{1}\right) \mid m_{a}\right),
$$

where $K_{a}=K\left(m_{a}\right)$. We set $z=z_{2}$ and $C_{s a}=\rho_{p}^{2}\left(z_{1}\right) / m_{a}$ in (21), and then insert (9) and (12) to obtain

$$
\mathrm{dn}^{2}\left(K_{a}-g_{a} / \sqrt{m_{a}} \mid m_{a}\right)=1-\frac{m_{b} R_{a}}{1-\left(1-R_{a}\right) m_{b}} .
$$

This can also be written, using (16) and the identity $\operatorname{dn}^{2}(\Lambda \mid m)=1-m \operatorname{sn}^{2}(\Lambda \mid m)$, as

$$
\operatorname{sn}^{2}\left(K_{a}-g_{a} / \sqrt{m_{a}} \mid m_{a}\right)=\sin ^{2} \phi,
$$

where

$$
\sin \phi=\sqrt{\frac{m_{b}\left(1-m_{a}\right)}{R_{b} m_{a}}}, \quad 0 \leq \phi \leq \frac{\pi}{2} .
$$

Note that $m_{a}$ is a constant when $R_{a}=1$. In this case, $m_{b}$ and $g_{b}$ are periodic functions of $g_{a}$. More generally we can invert (23) to obtain

$$
g_{a}=\sqrt{m_{a}}\left[J \mathrm{~F}\left(\phi \mid m_{a}\right)+\left(2 n_{a}-1\right) K_{a}\right],
$$

where $n_{a}$ is a positive integer, $J= \pm 1$, and $\mathrm{F}$ is the incomplete elliptic integral of the first kind. Note that (25) is identical to (43) of [1], where it was applied to an OPO crystal followed by an SFG crystal (the OPO $\rightarrow$ SFG configuration). Equations (16), (20), and (25) can be used to generate curves of complete pump depletion in the $\left(g_{a}, g_{b}\right)$ space, using $m_{b}$ as a generating parameter.

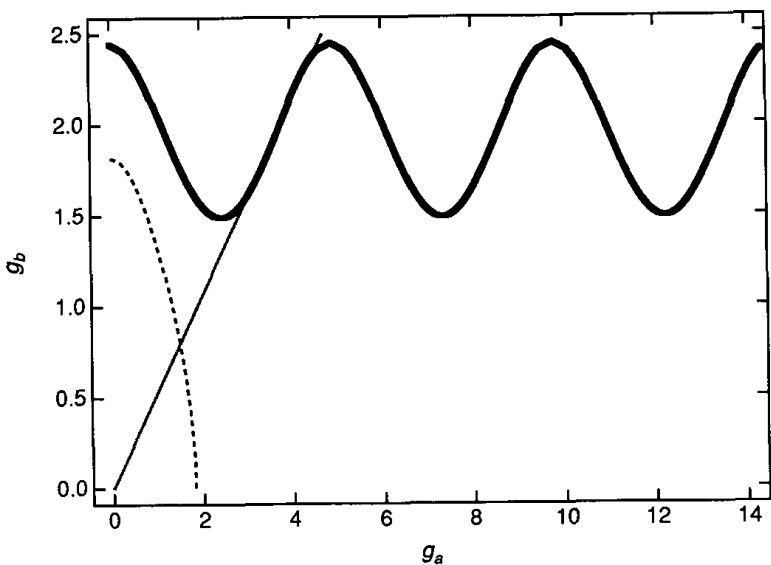

Fig. 4. Pump-depleted steady states for $n_{b}=1, R_{a}=1.0$, and $R_{b}=0.1$. These are all stable within the context of plane-wave theory. We here choose an operating line of slope 0.53 .

Now let us consider the stability of the steady states with complete pump depletion. This stability analysis is tractable if we restrict out treatment to the plane-wave model, though it cannot identify instabilities leading to the growth of additional temporal or transverse spatial frequencies. The analysis is nearly identical to that given in [1] for the OPO $\rightarrow$ SFG configuration, to which the reader should refer to details. We consider small perturbations $\delta_{j}(z)$ about the steady-state solutions, and define $\epsilon_{j}=\rho_{j} \delta_{j}$. The size $S$ of a perturbation $\epsilon_{s}\left(z_{1}\right)$ after a round-trip through the resonator relative to its original value is called the stability parameter. The steady states are unstable when $|S|>1$. At the end of the first crystal, the signal and pump perturbations are $\epsilon_{s}\left(z_{2}\right)=\epsilon_{s}\left(z_{1}\right)(1-\Delta)$ $[1,(54)]$ and $\epsilon_{p}\left(z_{2}\right)=\epsilon_{s}\left(z_{1}\right) \Delta[1,(74)]$, where $\Delta$ is given by $[1,(73)]$. At the entrance of the second crystal, we have $\epsilon_{s}\left(z_{3}\right)=R_{a} \epsilon_{s}\left(z_{1}\right)(1-\Delta)$, while $\epsilon_{p}\left(z_{3}\right)=\epsilon_{s}\left(z_{1}\right) \Delta$ is unchanged. Variation of the Manley-Rowe equation (7) shows that $\epsilon_{p}+\epsilon_{s}$ is constant over the second crystal. Because of the complete pump depletion, we have $\epsilon_{p}\left(z_{4}\right)=0$. Therefore, the signal perturbation is $\epsilon_{s}\left(z_{4}\right)=\epsilon_{s}\left(z_{1}\right)\left[1+\left(1-R_{a}\right) \Delta\right]$. Multiplying by $R_{b}$, we obtain the stability parameter

$$
S=R_{b}\left[1+\left(1-R_{a}\right) \Delta\right] .
$$

Equation (26) always predicts stable operation when outcoupling is only after the second crystal. We shall see later that this prediction is not borne out by a numerical simulation which incorporates transverse effects.

The plane-wave theory predicts a large dynamic range for high efficiency when there is large output coupling after either the first or the second OPO crystal. We consider two cases. In Fig. 3, we show the pump-depleted steady state for $n_{b}=1$, $R_{a}=0.1$, and $R_{b}=1.0$. The thick lines show the stable steady states. The filled circles show the zeros of $S$. The dashed line shows the small-signal threshold. The dotted line of slope $g_{b} / g_{a}=2.1$ lies close to the curve of complete pump depletion, and is our choice of operating line. Fig. 4 shows a similar plot for the case $n_{b}=1, R_{a}=1.0$, and $R_{b}=0.1$. In this case, we choose an operating line with slope $g_{b} / g_{a}=0.53$. 


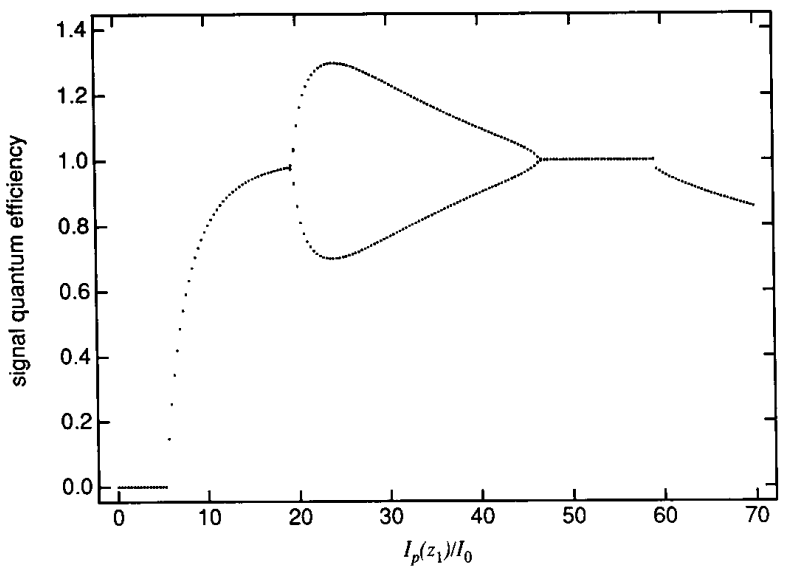

Fig. 5. Signal quantum efficiency for OPO-OPO device with outcoupling after first crystal is shown as a function of incident pump intensity, using the operating line in Fig. 3. A large domain of high-efficiency steady-state and limit-cycle operation is obtained. Intensity scale is the same in Figs. 5-9, if the same total effective crystal length is used. These plots show results for passes 113-128, after starting from $\hat{\rho}_{s}=0.01$ on the first pass.

\section{ItERATION OF Plane-Wave Solutions}

Further insight into the behavior of the OPO-OPO device can be obtained by iterating the plane-wave solutions of (1) - (3) over many passes through the resonator, starting with a small value of the signal. This calculation is repeated for a range of values of the pump intensity. We use $\left(g_{a}+g_{b}\right)^{2}=$ $I_{p}\left(z_{1}\right) / I_{0}$ as a dimensionless measure of the pump intensity, where $I_{0}=\frac{1}{2} c \epsilon_{0} \omega_{p} /\left(\kappa_{a} L_{a}+\kappa_{b} L_{b}\right)^{2}$. This scaling depends on the total effective crystal length $\kappa_{a} L_{a}+\kappa_{b} L_{b}$, which facilitates comparison of two-crystal devices with outcoupling after the first or second crystal and single-crystal devices. We also define dimensionless photon flux amplitudes $\hat{\rho}_{j}=$ $\left(\kappa_{a} L_{a}+\kappa_{b} L_{b}\right) \rho_{j}$.

Fig. 5 shows the signal quantum efficiency $\eta_{s a}=(1-$ $\left.R_{a}\right) \rho_{s}^{2}\left(z_{2}\right) / \rho_{p}^{2}\left(z_{1}\right)$ after saturation as a function of $I_{p}\left(z_{1}\right) / I_{0}$ along the operating line in Fig. 3. We find a large domain of high average efficiency, which includes strong periodtwo limit-cycle oscillations. The idler is produced with the same average efficiency, but with much smaller limit-cycle oscillations in the total idler power outcoupled at $z_{2}$ and $z_{4}$. Because $\kappa L$ is considerably larger for the second crystal, most of the small-signal gain occurs there. The total gain $G_{a} G_{b}$ is high, typically on the order of $10^{4}$ to $10^{5}$. Because of the high output coupling, the net gain $G$ is an order-of-magnitude less. However, after saturation most of the energy conversion occurs in the first crystal. Because of the high output coupling, the power loading in the first crystal is dominated by the incident pump power. The power loading in the second crystal is small after saturation.

The iterated plane-wave calculations can also be carried out for cases where one or both of the crystals is not perfectly phase matched. This procedure was used in [2]. If, for example, the first crystal has wave-vector mismatch $q_{a}$ and phase mismatch $Q_{a}=\frac{1}{2} q_{a} L_{a}=0.5$, while all other parameters remain as in Fig. 5 , then we obtain the results shown in

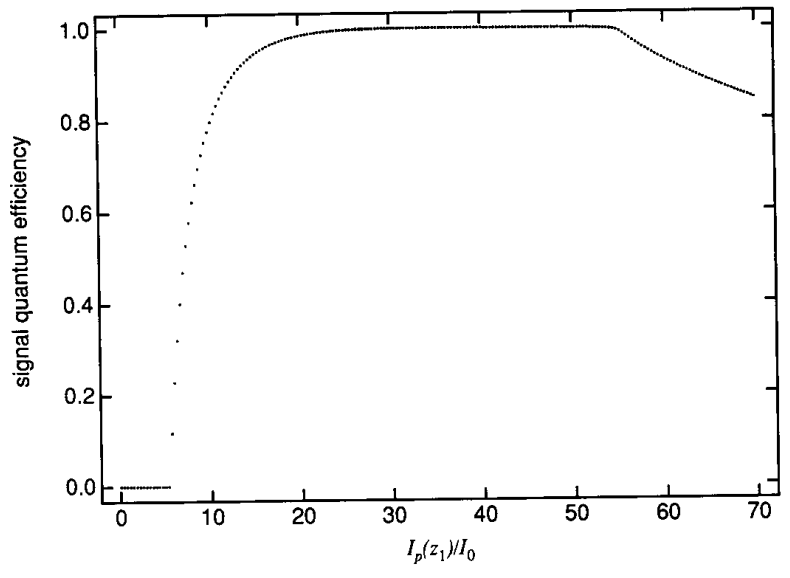

Fig. 6. Same as Fig. 5, except first crystal has a phase mismatch $Q_{a}=0.5$. The limit cycle is suppressed, but the efficiency remains high.



Fig. 7. Signal quantum efficiency for OPO-OPO device with outcoupling after the second crystal, using the operating line in Fig. 4 . The excellent efficiency found here using plane-wave theory is not borne out by calculations which include transverse effects.

Fig. 6. Here the limit cycle has been completely suppressed, while the average efficiency remains high. In practice the detunings $Q_{a}$ and $Q_{b}$ are not under complete experimental control, but vary with the signal temporal and transverse spatial frequencies. Nevertheless, we present numerical evidence below that detuning can suppress limit-cycle behavior, based on a model which includes transverse effects. For a typical high-efficiency point in Fig. 6, the flux densities are given by $\hat{\rho}_{p}^{2}\left(z_{1}\right)=28.83, \hat{\rho}_{p}^{2}\left(z_{2}\right)=\hat{\rho}_{p}^{2}\left(z_{3}\right)=4.17, \hat{\rho}_{p}^{2}\left(z_{4}\right)=$ $0.02, \hat{\rho}_{s}^{2}\left(z_{1}\right)=\hat{\rho}_{s}^{2}\left(z_{4}\right)=7.35, \hat{\rho}_{s}^{2}\left(z_{2}\right)=32.01, \hat{\rho}_{s}^{2}\left(z_{3}\right)=$ $3.20, \hat{\rho}_{i}^{2}\left(z_{2}\right)=24.66, \hat{\rho}_{i}^{2}\left(z_{4}\right)=4.15$. For type-II degenerate down-conversion at this point, $7 \%$ of the incident power is outcoupled in the idler polarization after the second crystal. Almost all the remaining power is outcoupled at the signal and idler frequency after the first crystal.

Fig. 7 shows the signal efficiency $\eta_{s b}=\left(1-R_{b}\right)$ $\rho_{s}^{2}\left(z_{4}\right) / \rho_{p}^{2}\left(z_{1}\right)$ for outcoupling after the second crystal using the operating line in Fig. 4 . We again find a large domain for 


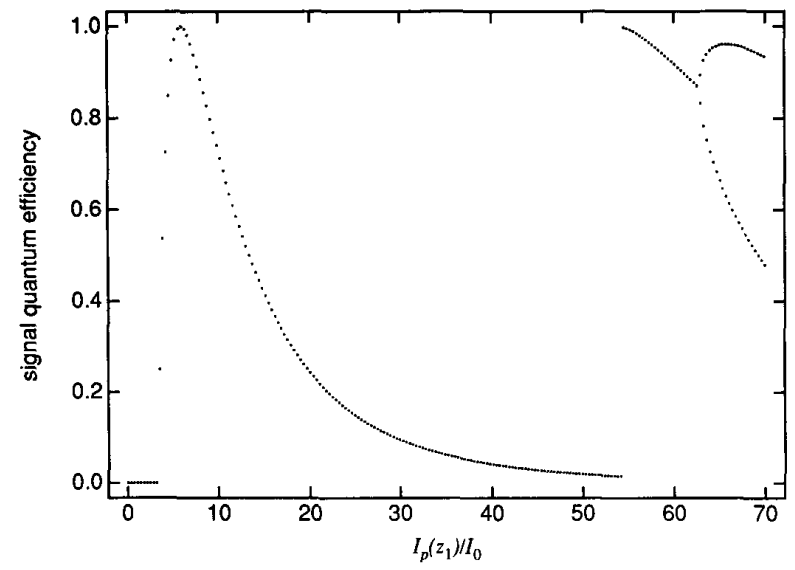

Fig. 8. Signal quantum efficiency for a single-crystal OPO with $90 \%$ output coupling.

high-efficiency conversion. Steady-state operation now occurs throughout this domain using phase-matched crystals, and high efficiency begins at a lower value of pump intensity. In this case the first crystal is longer than the second crystal, and both crystals are subject to high intensity, so the device is more nonlinear. Here, we define the degree of nonlinearity by the size of the quantities $\kappa_{a} L_{a} \rho_{j}(z)$ and $\kappa_{b} L_{b} \rho_{j}(z)$ at saturation. It turns out that transverse effects and multi-frequency instabilities can invalidate the plane-wave model, making the excellent performance predicted by Fig. 7 unrealizable. This configuration is also less advantageous for type-II degenerate down-conversion because more of the idler polarization is outcoupled at the position where the signal is not outcoupled. Energy conversion in the long crystal is greater at saturation in this configuration than in the configuration where the signal is outcoupled after the first crystal, though energy conversion in the short crystal is still dominant. For the example considered above where $\hat{\rho}_{p}^{2}\left(z_{1}\right)=28.83$, we find that $\hat{\rho}_{i}^{2}\left(z_{2}\right)=9.53$ and $\hat{\rho}_{i}^{2}\left(z_{4}\right)=19.30$.

Fig. 8 shows the signal efficiency for a single-crystal OPO with $90 \%$ output coupling and the same effective crystal length as the previous figures. This device has more small-signal gain than either of the two-crystal devices and is even more nonlinear than the device considered in Fig. 7. High efficiency occurs only for special values of the pump intensity.

Fig. 9 shows the signal quantum efficiency for the nonresonant device described earlier. Here, the two crystals are chosen to have equal effective length, corresponding to the situation in [3] and [4]. The signal is outcoupled after the first crystal, and the idler is outcoupled after the second crystal. The device behaves chaotically over most of the intensity range considered. Although on average the same number of signal and idler photons are generated, the signal quantum efficiency on a single pass can range up to $200 \%$, while the idler quantum efficiency is limited to $100 \%$. We have calculated the steady states with complete pump depletion for the nonresonant device, and find that no choice of operating line is possible which lies close to these states over an extended range.



Fig. 9. Signal quantum efficiency for the nonresonant two-crystal device with equal effective crystal lengths. This device displays largely chaotic behavior. The idler quantum efficiency is also largely chaotic, but is limited to $100 \%$ on any pass.

\section{Physical Example and Transverse Effects}

At this point, we extend our analysis of the OPO-OPO device beyond the plane-wave theory by means of a numerical model that incorporates transverse effects in one spatial dimension. For this purpose, it is useful to consider a specific example, namely degenerate down-conversion of $1.064 \mu \mathrm{m}$ radiation in the $X Z$ plane of KTP. To obtain the high gain characteristic of the OPO-OPO device, we propose synchronous pumping of the device with mode-locked, $Q$ switched ND:YAG radiation. Type-II phase matching occurs [5] at $53.94^{\circ}$ from the $Z$ axis. The pump is polarized in the $Y$ direction. One of the $2.128-\mu \mathrm{m}$ polarizations has a walk-off toward the $Z$ axis of $2.74^{\circ}$. Except where otherwise stated, we suppose that this is the resonant signal polarization, though we find equally good performance if the $Y$ - polarized light is resonant. We choose $L_{a}=0.476 \mathrm{~cm}$ and $L_{b}=$ $1.0 \mathrm{~cm}$, corresponding to the operating line in Fig. 3. Signal outcoupling with $R_{a}=0.1$ occurs after the first crystal. The crystal indices of refraction are $n_{s}=1.7719, n_{i}=1.7221$, and $n_{p}=1.7470$. The effective nonlinear coefficient is $d=$ $5.92 \mathrm{pm} / \mathrm{V}$. The characteristic intensity is therefore $I_{0}=$ 10.6 MW/ $/ \mathrm{cm}^{2}$. Let $x$ be one of the transverse dimensions orthogonal to the carrier wave-vector direction $z$. We suppose that the incident pump intensity is a real Gaussian in $x$ with a peak intensity $53.29 I_{0}=566 \mathrm{MW} / \mathrm{cm}^{2}$, which is near the high end of the high-efficiency range in Figs. 5 or 6 . The pump beam waist is thus at $z_{1}$. The peak intensity is below the KTP damage threshold of $1 \mathrm{GW} / \mathrm{cm}^{2}$, and the recirculating signal field increases the power loading only slightly. We take the $1 / e$ half-width of the incident pump amplitude to be $0.5 \mathrm{~mm}$.

We note that increasing the crystal lengths and the beam widths proportionally, for fixed pump power, would provide the same dynamical range for high-efficiency conversion at reduced peak intensity. This would give a greater safety margin to avoid damage to the crystals and other optical components. Although the available lengths of KTP crystals are limited, the crystal of length $L_{b}$ could be replaced by two shorter crystals 
in series. A phase-adjusting plate between these crystals might be needed to obtain constructive interference of the optical parametric interaction in the two crystals. This plate would compensate for phase shifts produced by anti-reflection coatings or the polar asymmetry of the KTP nonlinear coefficient [6].

Although our numerical model accounts for only one transverse dimension, we must check that the parameters we use are realistic when the time and both transverse dimensions are taken into account. For a round pump beam, the peak power is $P_{p}=(\pi / 2)\left(566 \mathrm{MW} / \mathrm{cm}^{2}\right)(0.5 \mathrm{~mm})^{2}=2.22 \mathrm{MW}$. Mode-locked pulses $T=100 \mathrm{psec}$ long thus have an energy of $2.22 \times 10^{-4} \mathrm{~J}$. With 20 mode-locked pulses per $Q$ switched macropulse and a $1 \mathrm{kHz} Q$-switched repetition rate, the average laser power is $4.44 \mathrm{~W}$. These parameters are easily obtained from commercial Nd:YAG lasers. With a noiseequivalent power $P_{\text {noise }}=\hbar \omega_{s} / T=9.3 \times 10^{-10} \mathrm{~W}$ and a net small-signal gain $G=3000$, we expect saturation to occur after $\ln \left(P_{p} / P_{\text {noise }}\right) / \ln G=4.4$ passes. One could obtain saturation in 2.7 passes by injection seeding with $1 \mathrm{~mW}$ at the signal frequency.

We describe the three-wave coupling in terms of slowly varying complex amplitudes $\sigma_{j}(z, x)$, where $\left|\sigma_{j}\right|=\rho_{j}$, modulating carrier plane waves propagating in the $z$ direction. Equation (1)-(3) are generalized to become

$$
\begin{gathered}
\frac{\partial \sigma_{s}}{\partial z}+\alpha_{s} \frac{\partial \sigma_{s}}{\partial_{x}}-\frac{i \lambda_{s}}{4 \pi n_{s}} \frac{\partial^{2} \sigma_{s}}{\partial x^{2}}=i \kappa \sigma_{p} \sigma_{i}^{*} e^{i q z}, \\
\frac{\partial \sigma_{i}}{\partial z}+\alpha_{i} \frac{\partial \sigma_{i}}{\partial x}-\frac{i \lambda_{i}}{4 \pi n_{i}} \frac{\partial^{2} \sigma_{i}}{\partial x^{2}}=i \kappa \sigma_{p} \sigma_{s}^{*} e^{i q z} \\
\frac{\partial \sigma_{p}}{\partial z}+\alpha_{p} \frac{\partial \sigma_{p}}{\partial x}-\frac{i \lambda_{p}}{4 \pi n_{p}} \frac{\partial^{2} \sigma_{p}}{\partial x^{2}}=i \kappa \sigma_{s} \sigma_{i} e^{-i q z}
\end{gathered}
$$

Here, $\alpha_{j}$ is the tangent of the walk-off angle, $\lambda_{j}$ is the freespace wavelength, and $q$ is the wave-vector mismatch for the carrier waves. The parameters $\alpha_{j}, n_{j}, \kappa$, and $q$ may be different for the two crystals. Here we assume that the nonzero $\alpha_{s}$ has opposite signs in the two crystals. The above equations can be converted into a rather complete description of the paraxial OPO interaction by including terms with first and second derivatives with respect to the other transverse coordinate $y$ and first (and possibly second) derivatives with respect to the time $t$. However, multipass calculations with more than one coordinate besides $z$ would be very slow and expensive, even on a supercomputer, unless the numerical step sizes were increased greatly. The diffractive terms containing second derivatives with respect to $x$ are approximate in that they neglect birefringence. This is not an important concern, since our two-dimensional model cannot accurately account for diffraction in three dimensions. Moreover, we iterate the solutions of (27)-(29) over a number (typically 20) of passes, without considering diffraction or focusing elements in the gaps between the crystals. We take the incident pump field to be the same on each pass, which is not the case for realistic $Q$-switched mode-locked pulse trains. Growth from the smallsignal regime and convergence to steady state (or limit cycle) behavior is rapid because of the high gain and large-output coupling. The numerical solution of $(27)-(29)$ utilizes the fast Fourier transform (FFT) with respect to $x$, combined with a fourth-order Runge-Kutta integration procedure applied to the discretized equations in the transverse-wave-vector domain. The calculations reported here use FFT's of size 512 with 16 Runge-Kutta steps in $z$ per crystal.

Fig. 10 shows the steady-state nonzero flux densities $\hat{\rho}_{j}^{2}$ as a function of $x$ at $z_{1}, z_{2}$, and $z_{4}$. These are shown in a frame moving with the signal rays, so that the pump and idler slip to the right by $0.228 \mathrm{~mm}$ in the first crystal and slip by $-0.478 \mathrm{~mm}$ in the second crystal. The detunings $Q_{a}$ and $Q_{b}$ are taken to be zero. The calculation also yields angular spectra (far-field distributions) for the various beams, as shown in Fig. 11. Saturation occurs in a few passes, starting from a Gaussian signal beam with peak $\hat{\rho}_{s}=10^{-5}$. In terms of fluxes obtained by integrating the flux densities over $x$, the gain is maximal on the second pass at $G_{a} G_{b}=(15.0)(2.08$ $\left.\times 10^{3}\right)=3.12 \times 10^{4}$. After saturation and convergence to steady state, we find that $88.8 \%$ of the incident pump energy is outcoupled at the signal frequency at $z_{2}$, of which $47.2 \%$ is in the signal polarization and $41.6 \%$ is in the idler polarization. Both polarizations display good beam quality in both the near and far fields. An additional 5.7\% of idler power (in a beam of poor quality) and $5.5 \%$ of unconverted pump are outcoupled at $z_{4}$.

A number of other examples have been studied using our numerical model. We describe some of the results briefly here without presenting figures. Nearly the same average efficiencies are obtained as above if the $Y$ - polarized light is resonated, but in this case a period-two limit cycle similar to Fig. 5 is obtained. The quantum efficiencies are $\eta_{s a}=$ $103.5 \%, \eta_{i a}=89.5 \%$, and $\eta_{i b}=2.6 \%$ on one pass and $\eta_{s a}=$ $82.6 \%, \eta_{i a}=77.7 \%$, and $\eta_{i b}=16.2 \%$ on the succeeding pass. The limit cycle can be suppressed by setting $Q_{a}=0.75$, as suggested by the results obtained in Fig. 6. In this case, steady-state behavior is obtained with essentially the same efficiency.

A calculation with zero detunings and zero walk-offs also gives a period-two limit cycle with about the same average efficiency, but with symmetrical and somewhat wider beam profiles and angular spectra. One could interpret this calculation as describing behavior in the transverse dimension perpendicular to walk-off, if the beams were wide in the walkoff direction. In this case, a detuning $Q_{a}=0.5$ is sufficient to suppress the limit-cycle oscillations, and the efficiency still remains about the same. It remains an open question under what conditions behavior approximating limit-cycle behavior would be observed with a round pump beam and with pulses within a $Q$ - switched envelope.

Now let us compare the above results with those obtained when the signal is outcoupled after the second crystal, using the operating line in Fig. 4 and the same total crystal length. The same pump beam is used. We assume zero detunings $Q_{a}$ and $Q_{b}$ and walk-off reversal in the two crystals. We find that the system appears close to a steady state at pass 20 , and that $56.9 \%$ of the incident pump energy is outcoupled at the signal frequency at $z_{4}$, of which $43.2 \%$ is in the 


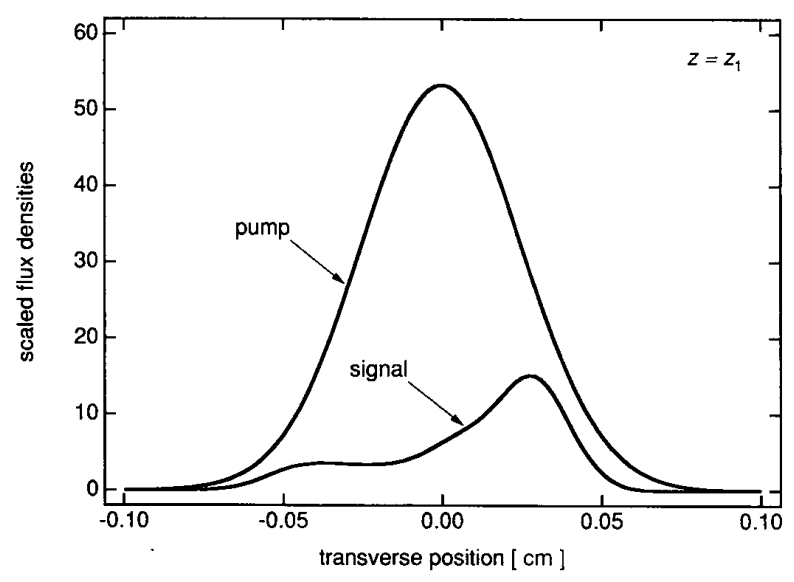

(a)

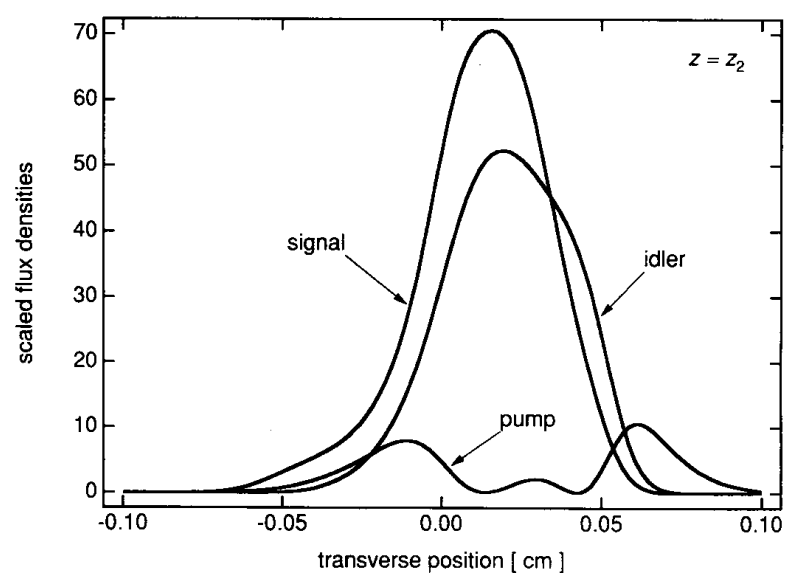

(b)

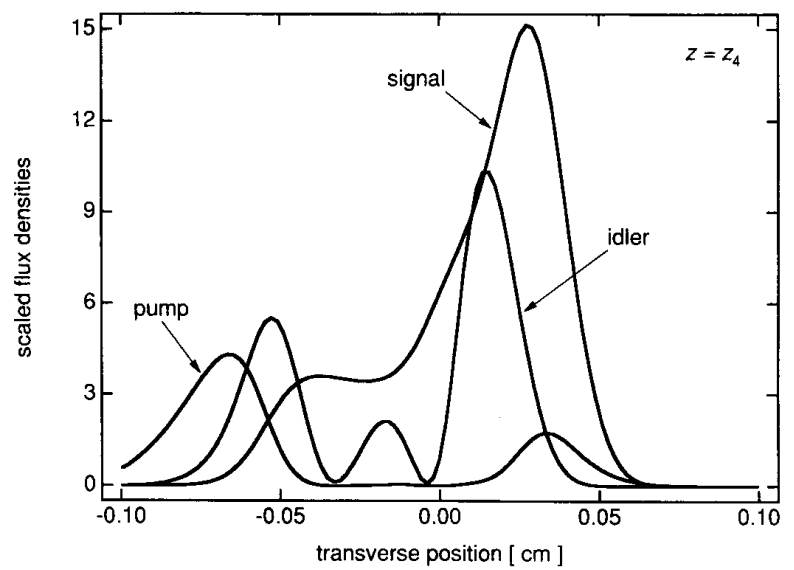

(c)

Fig. 10. Nonzero photon flux densities $\hat{\rho}_{j}^{2}=\left|\hat{\sigma}_{j}\right|^{2}$ are shown as a function of transverse position $x$ after 20 passes starting with a peak signal amplitude $\hat{\rho}_{s}\left(z_{1}\right)=10^{-5}$ on the first pass. Walk-off and diffraction are appropriate for type-II degenerate down-conversion of $1.064 \mu \mathrm{m}$ radiation in KTP. Outcoupling is after the first crystal. (a) $z=z_{1}$, (b) $z=z_{2}$, (c) $z=z_{4}$.

signal polarization and $13.7 \%$ is in the idler polarization. An additional $29.4 \%$ is outcoupled as idler at $z_{2}$, and $13.7 \%$

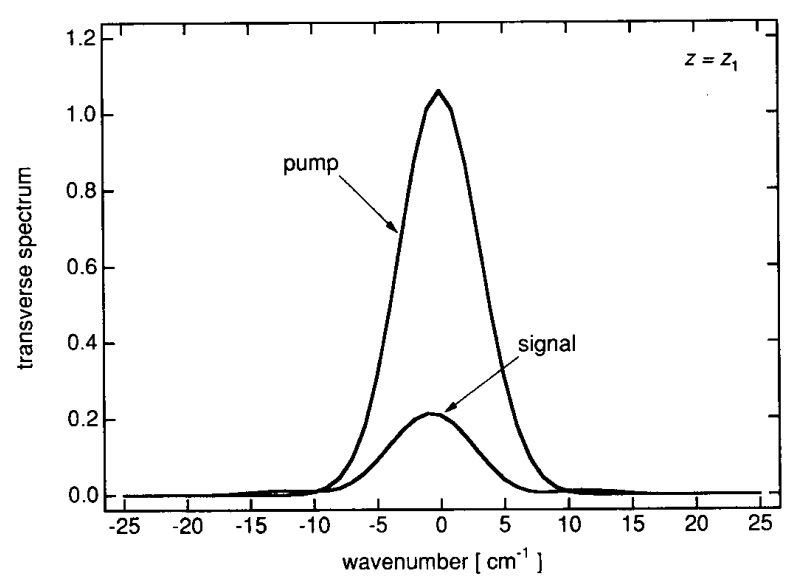

(a)



(b)



(c)

Fig. 11. Transverse angular spectra obtained by Fourier transforming the complex amplitudes $\hat{\sigma}_{j}$ and squaring are shown at (a) $z=z_{1}$, (b) $z=z_{2}$, (c) $z=z_{4}$.

of the pump is unconverted. This configuration gives much wider multipeaked angular spectra and sharp spikes in the $x$ domain. The angular spectra widen even more if smaller walk-offs are used, which can lead to numerical instabilities. 
Walk-off is associated with phase mismatch which limits the angular acceptance. In the transverse dimension perpendicular to the walk-off, only diffraction-produced phase mismatch and lack of spatial beam overlap limit the angular width, so that instabilities associated with this dimension may be more significant. Reducing the peak pump intensity to $36 I_{0}$ leads to even lower conversion efficiency ( $28 \%$ unconverted pump after 20 passes and increasing). In short, the optimistic results found in Fig. 7 are not borne out when transverse effects are taken into account. This configuration gives lower efficiency, poor beam quality, and is not so effective at concentrating most of the output into a single beam.

Next, we want to compare the OPO-OPO with a singlecrystal OPO of the same total crystal length pumped by the same beam as in Fig. 10. Here, the OPO appears close to a steady state after 20 passes. The angular spectrum is multipeaked and about three times as wide as that of the OPO-OPO, with complicated spiking in the $x$ domain. On pass 20 we find that $75.5 \%$ of the pump energy is outcoupled at the signal frequency, of which half is in the signal polarization and half is in the idler polarization. The remainder of the pump energy is unconverted. We again expect more unstable behavior for the direction perpendicular to walk-off.

To check that the single-crystal calculation just described is not biased unfavorably compared to the OPO-OPO by the fact that the latter used two crystals with walk-off reversal, we carried out a calculation where the single crystal just described was divided into two crystals half as long, with walk-off reversal but no output coupling or phase shifts between them. Again the device appears close to steady state after 20 passes. The energy conversion decreases to $65.9 \%$, with poor beam quality in both the near and far fields.

In conclusion, we find that the OPO-OPO device with outcoupling after the first crystal gives higher efficiency, stabler operation, and narrower angular spectra, and better beam quality than the conventional OPO or the OPO-OPO with outcoupling after the second crystal. We note that, when used for degenerate down-conversion, the output beam from any of these devices is formed by superposing the signal and idler polarizations. This output beam will not generally be well polarized. This is because temporal and transverse spatial frequency shifts for the signal and the idler are opposite to each other, and because the signal and idler beams have different spatial profiles.

\section{REFERENCES}

[1] G. T. Moore and K. Koch, "Optical parametric oscillation with intracavity sum-frequency generation," IEEE J. Quantum Electron., vol. 29, 961-969, 1993.

[2] _ , "Optical parametric oscillation with detuned intracavity sumfrequency generation," IEEE J. Quantum Electron., vol. 29, pp. 233-234, 1993.

[3] D. R. Guyer and D. D. Lowenthal, "Novel cavity design for a high efficiency, high energy near infrared $\beta-\mathrm{BaB}_{2} \mathrm{O}_{4}$ parametric generator," SPIE, Nonlinear Optics, vol. 1220, pp. 41-44, 1990.

[4] D. D. Lowenthal, "2-micron optical parametric sources," SPIE, SolidState Lasers IV, vol. 1864, 190-199, 1993.

[5] E. C. Cheung and J. M. Liu, "Efficient generation of ultrashort, wavelength-tunable infrared pulses," J. Opt. Soc. Am B, vol. 8, pp 1491-1506, 1991. Also in Data sheet No. 706, "Nonlinear optical properties of KTP," Quantum Technology, Inc., Lake Mary, FL.

[6] B. Ya Zel'dovich, Yu. E. Kapitskiii, and A. N. Chudinov, "Interference between second harmonics generated in two different KTP crystals," Sov. J. Quantum Electron., vol. 20, pp. 1120-1121, 1990.

Gerald T. Moore, for a biography, see p. 527 of the March issue of this JOURNAL.

Karl Koch, for a biography, see p. 527 of the March issue of this JouRnAL. 